\title{
MDM2 (RS769412) G>A Polymorphism in Cigarette Smokers: a Clue for the Susceptibility to Smoking and Lung Cancer Risk
}

\author{
Dilshad Ahmad, Abdul Karieem Bakairy,Abdull Malika Katheri, Waleed Tamimi
}

\begin{abstract}
Cigarette smoke contains oxidants and free radicals which are carcinogens that can induce mutations in humans. Single nucleotide polymorphisms (SNPs) are the most frequent genetic alterations found in the human genome. In the present study, we have examined the ability of the murine double minute 2 (Mdm2) (rs769412) A $>$ G polymorphism in cigarette smokers to predict risk of cancers. Our results showed that of smokers, $87 \%$ were found with AAgenotype, $10 \%$ with heterozygous AG genotype, and $3 \%$ with GG genotype. The heterozygous AG genotype was observed in a lower percentage of smokers (10\%) as compared to non-smokers $(18 \%)$, whereas, homozygous AA genotype was observed in lower percentage of non-smokers $(\mathbf{8 1 \%})$ as compared to the smokers $(87 \%)$. The results from present study support the association with an allele and AG genotype in non-smokers. However, further studies are required to establish the role of Mdm2 (rs769412) $\mathrm{C}>\mathrm{T}$ in cigarettes smokers and diseases.
\end{abstract}

Key words: Lung cancer - smoking risk - MDM2 polymorphism

Asian Pac J Cancer Prev, 16 (9), 4057-4060

\section{Introduction}

Cigarette smoking, even light or intermittent active or prolonged passive types are the biggest threat for many types of cancer, mainly of lung, and cardiovascular disease (CVD) (Bjartveit and Tverdal, 2005; Schane et al., 2010). According to the World Health Organization reports, approximately 1.3 billion people smoke worldwide (World Health Organization, 2008) and, consequently, 5.4 million people die per annum (World Health Organization, 2011). As estimation, by 2030 , over 8 million people will die annually due to smoking-related health problems. Cigarette Smoking causes about $90 \%$ of all lung cancer deaths (Doll et al., 1994). Lung cancer has the lowest survival rates of all cancers, and is the most common cause of the death in the UK (United Kingdom Lung Cancer Coalition., 2007). The survivors of smoking-related cancers are at high risk of cancers, espe $\neg$ cially in the head and neck and urinary tract. It is very well documented that smoking can increase the risk of over a dozen other cancers (American Cancer Society, 2012). In addition to loss of human lives, smoking causes great economic damage worldwide, especially in developing countries (World Health Organization, 2011).

The prevalence of smoking is high among young males in low-income countries. As far as the Kingdome of Saudi Arabia is concerned, the prevalence of current smoking among adolescent ranges from 12-29.8\%. Global Youth Tobacco Survey which was conducted by WHO (World Health Organization., 2011) reported that $34.5 \%$ senior secondary school students smoke sometime while $20 \%$ of them were regular smokers. Currently the frequency rate of smoking is in range of $2.4-37 \%$ among young adults. Concurrently, that was $11.6 \%$ and $25 \%$ among adults and elderly population (Bassiony, 2009). This phenomenon may be a direct result of various factors like urbanization, promotional marketing strategies of tobacco industries, westernization and misconception that associates smoking with maturity (World Health Organization, 2011).

Recently there was great interest shown among scientists to study the relationship of cancer with single nucleotide polymorphisms (SNPs) including p21, bcl2 or Mdm2 gene. Several studies have already been attempted in some of these genes. One of the candidates, the murine double minute (Mdm2) oncogene, which codes for Mdm2 protein, is a negative regulator of p53 tumor suppressor protein. Mdm2 protein is known for its functions as an E3 ubiquitin ligase that recognizes the $\mathrm{N}$-terminal transactivation domain (TAD) of the p53 tumor suppressor and as an inhibitor of p53 transcriptional activation (Oliner et al., 1992; Iwakuma and Lozano, 2003). Associations of different single nucleotide polymorphisms (SNPs) within genes with the predisposition of several diseases have been done and more researches are going in these directions. $\mathrm{Mdm} 2$ is one of the highly targeted and studied genes for their involvement in different types of cancers. Recent studies on Mdm2 have been found to be associated with the various types of cancers. For example, Mdm2 SNP309 is associated with more advanced esophageal squamous cell carcinoma (Hong et al., 2005). In one of the study, 
Mdm2 SNP309 G allele increases risk but the T-allele is associated with earlier onset age of sporadic breast cancers in the Chinese population [Lum et al., 2008) in another study, one SNP285C may neutralize the effect of one SNP309G allele (Knappskog and Lonning., 2011). The association of Mdm2 T309G polymorphism with bladder cancer is also documented (Onat et al., 2006). A study on promoter SNPs of Mdm2 revealed the lack of association with lung cancer in a Chinese population (Hu et al., 2006). In the study by Zhuo et al ( 2012) it was emphasized that Mdm2 polymorphism might be an important factor of lung cancer threat even for the population of never smokers.

Genetic predisposition to lung cancer is likely to be both polygenic and heterogeneous, conferred by low penetrance and variable arrangements of comparatively common variants (Shields and Harris, 2000; Zhou et al., 2005). Moreover, it is important to mention that the smoking-gene interactions are the key of lung cancer predisposition (Sasco et a., 2004). Hence, this study was undertaken to examine the association of Mdm2 variant rs769412 $(A>G)$ with the cigarette smoking behavior. The polymorphism of interest was chosen because that had been reported to be associated with the cancer of breast. Hence we thought of to test the hypothesis, if the same polymorphism may be associated with the smoking behavior and possible in turn with the smoking related diseases. The outcome of this study will allow us understand the relationship between smoking behavior and possible occurrences of smoking related cancers in Saudi population.

\section{Materials and Methods}

In this study, 300 smokers of age range 18-50 years $(33+3.5$ years) and 300 age matched nonsmokers were recruited. Samples were collected from local clinics. The approval of the study was taken by the Ethical-Committee. The volunteers were asked to submit their consent for the following study. The study groups were drawn from the central region of the Kingdom of Saudi Arabia.

\section{Sample collection}

The volunteer participated in this study were selected on the basis of number of cigarette the smoke daily. Those who smoked more than five cigarettes were regarded as smokers and those don not smoke any kind of tobacco product were regarded as non-smokers. The volunteers were recruited at the community not at hospitals. There were no female subject in this study because of the some local contrivances. About $5 \mathrm{~mL}$ of venous blood was drawn using sterile heparinized syringe from smokers and nonsmoker individuals for genotyping.

\section{Extraction of DNA}

Genomic DNA was extracted using QIAamp mini blood DNA extraction kit as per the instructions of the manufacturer.

\section{TaqMan genotyping}

Genotyping was carried out using TaqMan (Applied Biosystems) according to the manufacturer's instructions.
Primers and probes were supplied directly by Applied Biosystems as Assays-by-Design ${ }^{\mathrm{TM}}$. All assays were carried out in 96-well plates. Cases and controls were arranged in a chequer board pattern on each plate to ensure even treatment during the assay procedure and each plate included negative controls (with no DNA). Plates were read on the ABI Prism 7500 Fast using the Sequence Detection Software (Applied Biosystems) using the following conditions: $50^{\circ} \mathrm{C}$ for 2 minutes, $95^{\circ} \mathrm{C}$ for 10 minutes, and then 40 cycles of amplification $\left(92^{\circ} \mathrm{C}\right.$ denaturation for 15 seconds, $62^{\circ} \mathrm{C}$ annealing/extension for 60 seconds). Failed genotypes were repeated. Assays in which the genotypes of duplicate samples did not show $>95 \%$ concordance were discarded and replaced with alternative assays with the same tagging properties.

\section{Statistical methods}

For each SNP, deviation of genotype frequencies in cases and controls were compared by $\chi^{2}$ test for heterogeneity (two degrees of freedom) and test for trend (one degree of freedom), In order to evaluate the ethnicity specific effect. Genotype specific risks were estimated as odds ratio (ORs). Statistically significant differences were found between the number of smokers and non-smokers according to the genotype (Table1) .The results have been combined and the risk associated with each SNP was estimated by allelic, dominant and recessive OR and associated $95 \%$ confidence intervals (CI). ORs with $95 \%$ condense intervals (CIs) were calculated to assess the strength of the association between polymorphism and smoking behavior. We explored the association for co dominant model, dominant model, recessive model and allele versus allele, respectively. All statistical tests were based on two-sided probabilities using SPSS. We used a threshold of $\mathrm{p} \leq 0.05$.

\section{Results}

We analyzed the polymorphic status of Mdm2 (rs769412) (G>A) by Taman based Real Time PCR analysis. To the best of our knowledge, this is the rest study in the Saudi Arabian population, which examined the association of the Mdm2 gene polymorphism with

Table 1. Distribution of Mdm2 (rs769412). Genotypes and Allelic Frequencies of the Study Population

\begin{tabular}{lcccccc}
\hline & \multicolumn{5}{c}{ Genotypes } \\
\cline { 2 - 7 } & AA (n) & $\%$ & AG (n) & $\%$ & GG (n) & $\%$ \\
\hline Non-Smokers n (\%) & 243 & 81 & 54 & 18 & 3 & 1 \\
Smokers n (\%) & 261 & 87 & 30 & 10 & 9 & 3 \\
\hline$* \chi^{2}$ (2 d. f.) $=10.500 ; \mathrm{p}=0.005$ for genotypes
\end{tabular}

Table 2. Distribution of Mdm2 (rs769412) Allele Type and Allilic Frequencies

\begin{tabular}{lllcc}
\hline & \multicolumn{4}{c}{ Allelotypes } \\
\cline { 2 - 5 } & A (n) & a.f. & G (n) & a.f. \\
\hline Non-Smokers (n) & 540 & 0.9 & 60 & 0.1 \\
Smokers (n) & 552 & 0.92 & 48 & 0.08 \\
\hline
\end{tabular}


Table 3. Odds Ratio with $95 \%$ CI of Mdm2 (rs769412) Gene in Smokers

\begin{tabular}{llccc}
\hline Genotypes & & Odds Ratio & 95\%CI & p-value \\
\hline & AA versus AG & 0.517 & $0.320-0.835$ & 0.006 \\
& AA versus GG & 2.793 & $0.747-10.438$ & 0.112 \\
& AG versus GG & 5.4 & $1.357-21.481$ & 0.013 \\
Dominant Model & AA versus AG+GG & 0.637 & $0.409-0.992$ & 0.045 \\
Recessive Model & GG versus AG+GG & 0.228 & $0.058-0.896$ & 0.024 \\
Allelotypes & A/G & 0.783 & $0.526-1.165$ & 0.226 \\
\hline
\end{tabular}

cigarette smokers.

\section{Mdm2 Polymorphism}

As shown in Table1, the genotypic distributions in smokers were determined as $87 \%$ for the AA, $10 \%$ for the heterozygous AG status, and $3 \%$ for the GG, respectively. The homozygous GG genotype was seen only two percent lower in non-smokers $(1 \%)$ in comparison of smokers (3\%). Heterozygous AG genotype was seen in higher percentage of non-smokers $(18 \%)$ when compared to smokers (10\%). But homozygous dominant genotype AA was found in high percentage (87\%) in smokers then that of non-smokers $(81 \%)$. There was significant difference among AA, AG and GG genotypes of smokers and nonsmokers $\left(\chi^{2}=10.5, \mathrm{p}<0.005\right)$. Also, $A$ or $\mathrm{G}$ allele was not found to be a significant factor associated with smokers $\left(\chi^{2}=1.23, p=0.26\right)$ (Table 2). Hence, these groups were combined before further statistical analysis (Table 3 ). Data showed that AA versus AG, AG versus $G G$ and dominant model AA versus $\mathrm{AG}+\mathrm{GG}$ as well as recessive model $\mathrm{GG}$ versus AG+GG genotypes exhibited significant difference between smokers and non-smokers (OR, 0.517 (95\%CI 0.320-0.835), $\mathrm{p}=0.006$; OR 5.4 (95\% CI 1.357-21.481), $\mathrm{p}=0.01$; OR 0.637 (95\%CI 0.409-0.992), $\mathrm{p}=0.04$; OR 0.228 (95\% CI 0.058-0.896), $\mathrm{p}=0.02$ respectively). Other model AA versus GG did not showed any significant differences ( $\mathrm{p}=0.11$ ) (Table 3 ).

Frequency of A allele was 0.9 and 0.92 among nonsmokers and smokers respectively whereas, for $\mathrm{G}$ allele it was 0.1 and 0.08 among and non-smokers and smokers respectively. Neither the A allele nor the $G$ alleles were significantly associated with smokers. However a higher percentage of AA and lower percentage of AG genotypes in smokers suggests that transition mutation of A allele to $\mathrm{G}$ favor the smoking behavior. In other words this case a reverse mutation $\mathrm{G}>\mathrm{A}$ have a protective role against cigarette smoking and that seems to be a factor for $\mathrm{A}$ to $\mathrm{G}$ transition mutation and heterozygous condition at rs769412 in Mdm2 gene.

\section{Discussion}

Cigarette smoking (CS) continues to be a major health hazard, and it contributes significantly to cancer of the nasal cavities and paranasal sinuses, nasopharynx, stomach, liver, kidney (renal cell carcinoma) and uterine cervix, lung, oral cavity, pharynx, larynx, oesophagus, pancreas, urinary bladder, renal pelvis for adenocarcinoma of the oesophagus, and myeloid leukaemia (Sasco et al., 2004). Mainstream cigarette smoke comprises $8 \%$ of tar and $92 \%$ of gaseous components and the gas phase contains $>1015$ free radicals/puff (Pryor and Stone 1993). In cigarette smoke there are 55 carcinogens including polycyclic aromatic hydrocarbons, N-Nitrosamines, Heterocyclic aromatic amines, Aza-arenes and aromatic amines etc. that have been evaluated by the International Agency for Research on Cancer (IARC) (Hecht., 2012). The free radicals and other oxidants present in cigarette smoke are capable to oxidize the biomolecules including DNA and may result in consequential disorders such as altered gene expression and cancer (Pryor and Stone, 1993).

Most of the lung cancer is caused by the smoking. Lung cancer is the major cause of deaths in UK whereas, in USA the survival rate of lung cancer patients are as greater as $43 \%$. Lung cancer is primarily caused by tobacco smoking, but susceptibility is likely modified by common genetic variation. In response to many forms of cellular stress, including DNA damage, the p53 protein functions to induce cell cycle arrest, DNA repair, senescence, or apoptosis. Although cigarette smoking is the predominant cause of lung cancer, but it is not developed in all smokers (Liu et al., 2005). suggesting that particular individuals may be more susceptible to cigarette smoke. Familial aggregation studies have provided evidence for a genetic component to lung cancer risk (Schwartz and Ruckdeschel, 2006). Therefore, susceptibility to lung cancer may be due, in part, to inter individual genetic variation in the form of single nucleotide polymorphisms (SNP) or common allele variants.

Inactivation of the TP53 tumor suppressor gene is a frequent and early event in lung carcinogenesis. The p53 protein functions to induce growth arrest, DNA repair, senescence, and apoptosis in response to cellular stress, including DNA damage. Consistent with the p53 tumor suppressor functions, mutations in $\mathrm{p} 53$ are present in $>90 \%$ of small cell lung cancers and $>50 \%$ of nonsmall cell lung cancers (Mechanic et al., 2007). It well known fact, the Mdm2 is a negative regulator of $\mathrm{p} 53$, since when p53 levels are elevated, MDM2 transcription increases, binds with p53, and blocking its transcriptional activity and targeting it for degradation (Freedman and Levine.,1999).

Recently there has been great interest in studying the association of lung cancer with SNPs, since they are the most common sources of human genetic variation, and may contribute to an individual's susceptibility to cancer (Boersma et al., 2006). Mdm2 SNP354 (rs769412) leads to an $\mathrm{A} \rightarrow \mathrm{G}$ base change at codon 354 and creates a Sp1 binding site, but it does not cause an amino acid substitution. However, Mdm2 SNP354 (rs769412), are associated with breast cancer incidence and outcome 
(Rajaraman et al., 2007). In an African-American population study of Mdm2 SNP354 (rs769412) showed the lack of association between occurrence of lung cancer in smokers (Pine et al, 2006), but this was not confirmed in a more recent study in China (Ren et al., 2013). In addition, in one study, Rajaraman (2007) reported the protective role of Ex12+162A>G (rs769412) in case of glioma cancer.

There have been a few studies on rs769412 of Mdm2 gene for their association with lung cancer and smoking behavior. The present study analyzes for the first time the $\mathrm{Mdm} 2$ variant (rs769412) $\mathrm{A}>\mathrm{G}$ in Saudi Arabian smokers. Results indicated that Mdm2 (rs769412) A>G single nucleotide polymorphism was found significantly associated with non-smokers $(\mathrm{p}=0.005)$ (Table 1). Our findings are in consistence with the previous findings [26,27], but not supported by Pine et al., 2006).

However, the lack of the available literatures and the extensive studies on the variation of Mdm2 (rs769412) $\mathrm{A}>\mathrm{G}$ and their association with the lung cancer and smoking behavior jeopardizes the supports to our finding. Hence, there is a great need of study the variation in different populations for different disease. Nevertheless, this study may be useful for the further researches on Mdm2 SNPs in smokers and their associated diseases.

\section{References}

American Cancer Society (2012). Cancer treatment and survivorship facts \& figures 2012-2013. Atlanta.

Bassiony MM (2009). Smoking in Saudi Arabia. Saudi Medical $J$, 30, 876-81.

Bjartveit K, Tverdal A (2005). Health consequences of smoking 1-4 cigarettes per day. Tob Control, 14, 315-20.

Boersma BJ, Howe TM, Goodman JE (2006). Association of breast cancer outcome with status of p53 and MDM2 SNP309. J Natl Cancer Inst, 98, 911-9.

Doll R, Peto R, Wheatley K, et al (1994). Mortality in relation to smoking: 40 years' observations on male British doctors. BMJ, 309, 901-11.

Freedman DA, Levine AJ (1999). Regulation of the 533 protein by the MDM2 oncoprotein: Thirty-Eighth GHA Clowes Memorial Award Lecture. Cancer Res, 59, 1-7.

Hecht SS (2012). Lung carcinogenesis by tobacco smoke, Int $J$ Cancer, 131, 2724-32.

Hong Y, Miao X, Zhang X (2005). The role of P53 and MDM2 polymorphisms in the risk of esophageal squamous cell carcinoma. Cancer Res, 15, 9582-587.

$\mathrm{Hu} \mathrm{Z}$, Ma H, Lu D (2006). Genetic variants in the MDM2 promoter and lung cancer risk in a Chinese population. Int J Cancer, 1, 1275-278.

Iwakuma T, Lozano G (2003). MDM2, an introduction. Mol Cancer Res, 1, 993-1000

Knappskog S, Lonning PE (2011). Effects of the MDM2 promoter SNP285 and SNP309 on Sp1 transcription factor binding and cancer risk. Transcription, 11, 207-10.

Liu G, Zhou W, Christiani DC (2005). Molecular epidemiology of non-small cell lung cancer. Semin Respir Crit Care Med, 26, 265-72.

Lum SS, Chua HW, Li H et al (2008). MDM2 SNP309 G allele increases risk but the $\mathrm{T}$ allele is associated with earlier onset age of sporadic breast cancers in the Chinese population. Carcinogenesis, 29, 754-61.

Mechanic LE, Bowman ED, Welsh JA et al ( 2007). Common genetic variation in TP53 is associated with lung cancer risk and prognosis in African Americans and somatic mutations in lung tumors. Cancer Epidemiol Biomarkers Prev, 16, 214-22.

Oliner JD, Kinzler KW, Meltzer PS et al (1992). Amplification of a gene encoding a 53 -associated protein in human sarcomas. Nature, 358, 80-83.

Onat OE, Tez M, Ozçelik T (2006). MDM2 T309G polymorphism is associated with bladder cancer. Anticancer Res, 26, 3473475.

Pine SR, Mechanic LE, Bowman ED, et al (2006). MDM2 SNP309 and SNP354 are not associated with lung cancer risk. Cancer Epidemiol Biomarkers Prev, 15, 1559-561.

Pryor WA, Stone, K (1993). Oxidants in cigarette smoke. Radicals, hydrogen peroxide, peroxynitrate, and peroxynitrite. Ann $N$ $Y$ Acad Sci, 686, 12-27.

Zhou W, Liu G, Park S(2005). Gene-smoking interaction associations for the ERRC1 polymorphisms in the risk of lung cancer. Cancer Epidemiol Biomarkers Prev, 14, 491-6.

Rajaraman P, Wang SS, Rothman N et al (2007). Polymorphisms in apoptosis and cell cycle control genes and risk of brain tumors in adults. Cancer Epidemiol Biomarkers Prev, 16, 1655-61.

Ren YW, Yin ZH, Wan Y, et al (2013). P53 Arg72Pro and MDM2 SNP309 polymorphisms cooperate to increase lung adenocarcinoma risk in Chinese female non-smokers: a case control study. Asian Pac J Cancer Prev, 14, 5415-20.

Sasco AJ, Secretan MB, Straif K (2004). Tobacco smoking and cancer: a brief review of recent epidemiological evidence. Lung Cancer, 2, 3-9.

Schane RE, Ling PM, Glantz SA (2010). Review health effects of light and intermittent smoking. A review. Circulation. 121, 1518-22.

Schwartz AG, Ruckdeschel JC (2006). Familial lung cancer: genetic susceptibility and relationship to chronic obstructive pulmonary disease. Am J Respir Crit Care Med, 173, 16-22.

Shields P, Harris C (2000). Cancer risk and low-penetrance susceptibility genes in gene- environment interactions. $J$ Clin Oncol, 18, 2309-15.

United Kingdom Lung Cancer Coalition, Lung Cancer Plan (2007). Improving lung cancer survival in the UK, UK Lung Cancer Coalition November.

World Health Organization (2008). Report on the global tobacco epidemic, 2008-The MPOWER package, Geneva.

World Health Organization (2011). Report on the global tobacco epidemic (2011). Warning about the dangers of tobacco. Geneva: Switzerland.

Zhuo W,Zhang L, Zhu B (2012). Association of MDM2 SNP309 variation with lung cancer risk: evidence from 7196 cases and 8456 controls. PLOS ONE. 7, 41546. 